\title{
Genetic Diversity and Discrimination of Chimonanthus praecox (L.) Link Germplasm Using ISSR and RAPD Markers
}

\author{
Kai-Ge Zhao, Ming-Qin Zhou, and Long-Qing Chen ${ }^{1}$ \\ Key Laboratory of Horticultural Plant Biology, Ministry of Education, \\ College of Horticulture and Forestry Sciences, Huazhong Agricultural \\ University, Shizishan Street, Wuhan 430070, China
}

\section{Donglin Zhang}

Department of Plant, Soil, and Environmental Sciences, University of Maine, Orono, ME 04469-5722

\section{Gituru Wahiti Robert \\ Botany Department, Jomo Kenyatta University of Agriculture and Technology, PO Box 62000-0200 Nairobi, Kenya}

Additional index words. molecular marker, wintersweet, clone, genetic variation, germplasm resources, AMOVA

\begin{abstract}
Chimonanthus praecox (wintersweet) is endemic to China. It has been cultivated there for more than $\mathbf{1 0 0 0}$ years as a garden, potted, and cut-flower plant. Many cultivars have been developed during its long history of cultivation, and recently many germplasms were collected in Wuhan and Nanjing, China. The identification and genetic relationship of these resources were studied based mainly on morphological traits. In the current study, intersimple sequence repeat markers (ISSR) and random amplified polymorphic DNA markers (RAPD) were used for the first time to investigate 72 wintersweet clones from the two regions. Eleven ISSR primers amplified 115 bands, 90 (78.26\%) of which were polymorphic. Nineteen RAPD primers amplified 165 bands, 105 $(63.63 \%)$ of which were polymorphic. Either ISSR or RAPD markers were sufficient to distinguish all the clones surveyed. A Dendrogram based on Jaccard's similarity coefficients indicated that the distribution pattern of the 72 clones was coherent with their geographical origins. Most of the genetic variation $(85.68 \%$ with ISSR data; $86.75 \%$ with RAPD data) occurred among clones within each region. However, the difference between Wuhan and Nanjing groups is statistically significant $\left(\Phi_{\mathrm{ST}}=0.143, P<0.001\right.$, with ISSR data; $\Phi_{\mathrm{ST}}=0.132, P<0.001$, with $\mathrm{RAPD}$ data). Morphological variation and classification of wintersweet cultivars were also discussed compared with the genetic relationship based on ISSR and RAPD markers. This is the first report of the partitioning of genetic variability within and between different cultivated wintersweet regions, and it provides useful baseline data for optimizing sampling strategies in breeding. These results are important for future genetic improvement, identification, and conservation of Chimonanthus praecox germplasm.
\end{abstract}

Chimonanthus praecox (L.) Link, wintersweet, belongs to Calycanthaceae. It is a deciduous shrub and blossoms in winter from late November to March. Its flowers are

Received for publication 6 Jan. 2007. Accepted for publication 7 Mar. 2007.

The study was supported by the National Natural Science Foundation of China (no. 39900118) and International Foundation for Science (project no. D/2614-1)

Donglin Zhang is also a guest professor at Central South Forestry University, Changsha, Hunan Province 410004, China.

Maine Agricultural and Forestry Expt. Sta. publication 2941.

We thank the Mei Garden (Wuhan) and the Garden of Ming Xiao Ling (Nanjing) for providing some of the wintersweet samples, and Xian-Bao Zen and Xue-Lan Liu for their field assistance.

${ }^{1}$ To whom reprint requests should be addressed; e-mail chenlq0206@163.com been cultivated for more than 1000 years as an ornamental plant. It was introduced to Korea between 1611 and 1628 (Feng et al., 1990), then to Japan, Europe, America, and Australia.

After a long history of cultivation, a lot of wintersweet cultivars were developed, and many germplasms were collected in Wuhan and Nanjing, China, in recent years. Accurate identification and correct nomenclature of these cultivars are essential for the management of wintersweet germplasm resources. However, only a few cultivars were named, and some cases of homonymy and synonymy exist. The genetic information of wintersweet germplasm is also poorly understood. Although biochemical markers and morphological traits had been used in attempts to understand their genetic diversity (Chen, 1995; Zhao et al., 2004), the validity of the results obtained from such studies is considerably affected by the sensitivity of these traits to environmental factors and physiological stages of plants.

Polymerase chain reaction (PCR)-based DNA marker technologies are useful tools to identify closely related plants and to evaluate the genetic homogeneity of cultivars (Siragusa et al., 2006). Among the various molecular marker techniques, intersimple sequence repeat markers (ISSR) and random amplified polymorphic DNA markers (RAPD) are two commonly used methods, mainly because they are simple to execute, are low cost, and require neither sequence information nor prior genetic studies (Williams et al., 1990; Zietkiewicz et al., 1994). Many recent papers have demonstrated the high potential of these two markers in identification (Belaj et al., 2004), classification (Suo et al., 2005), and diversity analysis (MirAli and Nabulsi, 2003) of germplasm resources in agricultural crops. The objectives of this study were to set up a molecular profile that would allow a precise identification of wintersweet germplasm and to evaluate their genetic diversity and relationship based on these two markers.

\section{Materials and Methods}

Plant materials and DNA extraction. A total of 72 wintersweet clones (Table 1) were collected in this study. (In this paper, cultivars and genotypes are represented as "clones" because all accessions were propagated asexually.) Among them, 29 clones were from Nanjing (lat. $32^{\circ} \mathrm{N}$, long. $119^{\circ} \mathrm{E}$ ), and the other 43 clones were from Wuhan (lat. $30^{\circ} \mathrm{N}$, long. $114^{\circ} \mathrm{E}$ ). In these two cities, wintersweets have been widely planted in gardens for centuries. All collected clones had distinguished morphological characteristics through 3 years of observation.

Genomic DNA for both ISSR and RAPD analyses was extracted from young leaves following the sodium dodecyl sulfate (SDS) method described by Chen et al. (1999). DNA concentrations were quantified by a GeneRay ultraviolet photometer (Biometra, Göttingen, Germany), adjusted to $20 \mathrm{ng} \mathrm{uL}^{-1}$ for each PCR amplification. 


\begin{tabular}{|c|c|c|c|c|c|c|c|}
\hline Clone & Original name & Origin & $\begin{array}{c}\text { Color of inner } \\
\text { tepals }\end{array}$ & $\begin{array}{c}\text { Color of } \\
\text { medium tepals }\end{array}$ & $\begin{array}{c}\text { Shape of } \\
\text { medium tepals }\end{array}$ & Flower size ${ }^{y}$ & Flower shape \\
\hline$\overline{\mathrm{H} 1}$ & Unidentified & Wuhan & $\mathrm{C} 2$ & Yellow & Spoon shaped & Medium & Bowl shaped \\
\hline $\mathrm{H} 2$ & Zhaoxia & Wuhan & $\mathrm{C} 1$ & Yellow & Crimped & Big & Plate shaped \\
\hline H5 & Zhaoxia & Wuhan & $\mathrm{C} 1$ & Yellow & Crimped & Big & Plate shaped \\
\hline $\mathrm{H} 8$ & Jinhe Ziwen & Wuhan & $\mathrm{C} 2$ & Yellow & Strip shaped & Medium & Bell shaped \\
\hline H9 & Unidentified & Wuhan & $\mathrm{C} 2$ & Yellow & Strip shaped & Medium & Bell shaped \\
\hline $\mathrm{H} 10$ & Unidentified & Wuhan & $\mathrm{C} 3$ & Yellow & Spoon shaped & Medium & Bell shaped \\
\hline H11 & Unidentified & Wuhan & $\mathrm{C} 1$ & Whitish yellow & Strip shaped & Medium & Columnar \\
\hline H13 & Acuticoncolor & Wuhan & $\mathrm{C} 1$ & Golden & Strip shaped & Medium & Bell shaped \\
\hline $\mathrm{H} 21$ & Unidentified & Wuhan & $\mathrm{C} 2$ & Yellow & Strip shaped & Big & Plate shaped \\
\hline $\mathrm{H} 22$ & Unidentified & Wuhan & $\mathrm{C} 1$ & Golden & Strip shaped & Medium & Bell shaped \\
\hline $\mathrm{H} 25$ & Xuhe & Wuhan & $\mathrm{C} 1$ & Yellow & Spoon shaped & Medium & Bell shaped \\
\hline $\mathrm{H} 29$ & Unidentified & Wuhan & $\mathrm{C} 4$ & Yellow & Strip shaped & Medium & Bell shaped \\
\hline $\mathrm{H} 32$ & Unidentified & Wuhan & $\mathrm{C} 3$ & Yellow & Strip shaped & Medium & Columnar \\
\hline H33 & Unidentified & Wuhan & $\mathrm{C} 2$ & Greenish yellow & Crimped & Medium & Plate shaped \\
\hline H34 & Unidentified & Wuhan & $\mathrm{C} 4$ & Whitish yellow & Strip shaped & Medium & Bell shaped \\
\hline H35 & Unidentified & Wuhan & $\mathrm{C} 2$ & Yellow & Spoon shaped & Medium & Bowl shaped \\
\hline H36 & Huangmo & Wuhan & $\mathrm{C} 4$ & Yellow & Strip shaped & Medium & Bell shaped \\
\hline H37 & Elü & Wuhan & $\mathrm{C} 2$ & Greenish yellow & Crimped & Medium & Plate shaped \\
\hline H38 & Unidentified & Wuhan & $\mathrm{C} 2$ & Greenish yellow & Crimped & Medium & Plate shaped \\
\hline H39 & Unidentified & Wuhan & $\mathrm{C} 3$ & Golden & Strip shaped & Medium & Plate shaped \\
\hline H44 & Ceraceus & Wuhan & $\mathrm{C} 1$ & Yellow & Strip shaped & Medium & Bell shaped \\
\hline $\mathrm{H} 45$ & Unidentified & Wuhan & $\mathrm{C} 4$ & Whitish yellow & Strip shaped & Medium & Bell shaped \\
\hline $\mathrm{H} 46$ & Unidentified & Wuhan & $\mathrm{C} 4$ & Whitish yellow & Strip shaped & Small & Bell shaped \\
\hline H64 & Unidentified & Wuhan & $\mathrm{C} 1$ & Yellow & Oval & Medium & Bowl shaped \\
\hline H68 & Acuticoncolor & Wuhan & $\mathrm{C} 1$ & Golden & Strip shaped & Medium & Bell shaped \\
\hline H81 & Xuezi & Wuhan & $\mathrm{C} 4$ & Whitish yellow & Strip shaped & Medium & Bell shaped \\
\hline $\mathrm{H} 83$ & Bingyüzi & Wuhan & $\mathrm{C} 3$ & Whitish yellow & Oval & Big & Bell shaped \\
\hline H87 & Unidentified & Wuhan & $\mathrm{C} 3$ & Whitish yellow & Strip shaped & Medium & Columnar \\
\hline H89 & Unidentified & Wuhan & $\mathrm{C} 3$ & Yellow & Strip shaped & Medium & Bell shaped \\
\hline H90 & Jinlong Zixue & Wuhan & $\mathrm{C} 4$ & Yellow & Strip shaped & Medium & Plate shaped \\
\hline H94 & Unidentified & Wuhan & $\mathrm{C} 3$ & Yellow & Crimped & Medium & Plate shaped \\
\hline H97 & Unidentified & Wuhan & $\mathrm{C} 1$ & Yellow & Strip shaped & Medium & Bell shaped \\
\hline HY & Unidentified & Wuhan & $\mathrm{C} 2$ & Greenish yellow & Spoon shaped & Small & Urn shaped \\
\hline H46M & Unidentified & Wuhan & $\mathrm{C} 4$ & Whitish yellow & Oval & Big & Bowl shaped \\
\hline H48M & Unidentified & Wuhan & $\mathrm{C} 4$ & Yellow & Oval & Medium & Bowl shaped \\
\hline HG3 & Unidentified & Wuhan & $\mathrm{C} 2$ & Yellow & Strip shaped & Big & Plate shaped \\
\hline Y72 & Unidentified & Wuhan & $\mathrm{C} 2$ & Golden & Oval & Big & Bowl shaped \\
\hline W1 & Unidentified & Wuhan & $\mathrm{C} 3$ & Yellow & Strip shaped & Medium & Bell shaped \\
\hline M1 & Zixin Qingkou & Wuhan & $\mathrm{C} 4$ & Yellow & Spoon shaped & Small & Urn shaped \\
\hline M2 & Jinpan Zifeng & Wuhan & $\mathrm{C} 4$ & Yellow & Strip shaped & Big & Bell shaped \\
\hline M6 & Yanyan & Wuhan & $\mathrm{C} 1$ & Yellow & Spoon shaped & Big & Bell shaped \\
\hline M11 & Unidentified & Wuhan & $\mathrm{C} 1$ & Yellow & Strip shaped & Big & Plate shaped \\
\hline M22 & Bingzhu & Wuhan & $\mathrm{C} 1$ & Whitish yellow & Oval & Medium & Urn shaped \\
\hline N1 & Huangzijuan & Nanjing & $\mathrm{C} 3$ & Yellow & Strip shaped & Medium & Bell shaped \\
\hline $\mathrm{N} 2$ & Unidentified & Nanjing & $\mathrm{C} 3$ & Yellow & Strip shaped & Medium & Bell shaped \\
\hline N3 & Yangzhouhuang & Nanjing & $\mathrm{C} 1$ & Golden & Oval & Medium & Bowl shaped \\
\hline N4 & Unidentified & Nanjing & $\mathrm{C} 3$ & Whitish yellow & Spoon shaped & Medium & Columnar \\
\hline N5 & Jinlü & Nanjing & $\mathrm{C} 4$ & Yellow & Strip shaped & Medium & Bell shaped \\
\hline N6 & Unidentified & Nanjing & $\mathrm{C} 2$ & Yellow & Strip shaped & Medium & Bell shaped \\
\hline N10 & Jinguan & Nanjing & $\mathrm{C} 2$ & Golden & Strip shaped & Medium & Bell shaped \\
\hline N11 & Unidentified & Nanjing & $\mathrm{C} 2$ & Yellow & Strip shaped & Small & Bell shaped \\
\hline N12 & Unidentified & Nanjing & $\mathrm{C} 1$ & Yellow & Oval & Big & Bowl shaped \\
\hline N13 & Unidentified & Nanjing & $\mathrm{C} 4$ & Golden & Oval & Medium & Bowl shaped \\
\hline N15 & Unidentified & Nanjing & $\mathrm{C} 2$ & Yellow & Strip shaped & Medium & Bell shaped \\
\hline N16 & Unidentified & Nanjing & $\mathrm{C} 2$ & Whitish yellow & Oval & Medium & Bowl shaped \\
\hline N17 & Suhe & Nanjing & $\mathrm{C} 1$ & Whitish yellow & Oval & Medium & Bowl shaped \\
\hline N18 & Riguang & Nanjing & $\mathrm{C} 2$ & Yellow & Strip shaped & Medium & Bell shaped \\
\hline N19 & Unidentified & Nanjing & $\mathrm{C} 2$ & Golden & Oval & Medium & Bell shaped \\
\hline $\mathrm{N} 20$ & Jinghuang & Nanjing & $\mathrm{C} 1$ & Yellow & Strip shaped & Big & Bell shaped \\
\hline $\mathrm{N} 22$ & Qingkou Yinhong & Nanjing & $\mathrm{C} 3$ & Whitish yellow & Oval & Big & Urn shaped \\
\hline $\mathrm{N} 23$ & Unidentified & Nanjing & $\mathrm{C} 4$ & Yellow & Crimped & Big & Plate shaped \\
\hline N24 & Unidentified & Nanjing & $\mathrm{C} 1$ & Yellow & Spoon shaped & Small & Bell shaped \\
\hline $\mathrm{N} 25$ & Unidentified & Nanjing & $\mathrm{C} 4$ & Golden & Strip shaped & Small & Bell shaped \\
\hline $\mathrm{N} 28$ & Nanjing Qingkou & Nanjing & $\mathrm{C} 3$ & Yellow & Spoon shaped & Medium & Bell shaped \\
\hline N36 & Yüdie & Nanjing & $\mathrm{C} 1$ & Yellow & Strip shaped & Big & Bell shaped \\
\hline N37 & Jiangnanbai & Nanjing & $\mathrm{C} 1$ & Whitish yellow & Oval & Medium & Urn shaped \\
\hline N39 & Unidentified & Nanjing & $\mathrm{C} 3$ & Yellow & Oval & Big & Urn shaped \\
\hline N51 & Unidentified & Nanjing & $\mathrm{C} 4$ & Golden & Strip shaped & Medium & Bell shaped \\
\hline N55 & Nanjing Jinlian & Nanjing & $\mathrm{C} 1$ & Yellow & Strip shaped & Small & Bell shaped \\
\hline N56 & Unidentified & Nanjing & $\mathrm{C} 1$ & Golden & Strip shaped & Medium & Bell shaped \\
\hline N57 & Unidentified & Nanjing & $\mathrm{C} 1$ & Yellow & Strip shaped & Medium & Bell shaped \\
\hline N66 & Unidentified & Nanjing & $\mathrm{C} 4$ & Yellow & Oval & Small & Bowl shaped \\
\hline
\end{tabular}

${ }^{\mathrm{z}} \mathrm{C} 1$, yellow; $\mathrm{C} 2$, yellow sprinkled with a few mauve speckles; C3, yellow sprinkled with mauve blotches; C4, mauve.

${ }^{y}$ Flower size was described by Chen et al. (2004). 
Intersimple sequence repeat marker and $R A P D$ amplifications. Intersimple sequence repeat marker amplification was performed in a $25-\mu \mathrm{L}$ reaction volume, containing $40 \mathrm{ng}$ of template DNA, 1× PCR buffer (MBI Fermentas, Lithuania), $2 \mathrm{~mm} \mathrm{MgCl}_{2}, 0.2$ mM each of dNTP, $0.2 \mu \mathrm{M}$ ISSR primer (Sangon, Shanghai, China), 2\% formamide (Sigma, St. Louis), and 1 unit of Taq DNA polymerase (MBI Fermentas). All PCR reactions were run on a PE 9600 Thermal Cycler (Perkin Elmer Corp., Waltham, MA) using the following reaction condition: $5 \mathrm{~min}$ at $94{ }^{\circ} \mathrm{C}, 38$ cycles of $30 \mathrm{~s}$ at $94{ }^{\circ} \mathrm{C}, 45 \mathrm{~s}$ at 51 to $53{ }^{\circ} \mathrm{C}$, and $90 \mathrm{~s}$ at $72{ }^{\circ} \mathrm{C}$, with a final extension of $7 \mathrm{~min}$ at $72{ }^{\circ} \mathrm{C}$. Polymerase chain reaction products were separated on $2 \%(\mathrm{w} / \mathrm{v})$ agarose gels containing $0.5 \mu \mathrm{g} \cdot \mathrm{mL}^{-1}$ ethidium bromide in $1 \times \mathrm{TAE}$ buffer, and were photographed under ultraviolet light using a Gel-Logic 200 image system (EastmanKodak, Rochester, NY).

Random amplified polymorphic DNA marker amplifications were carried out in a 20- $\mu \mathrm{L}$ reaction volume containing $20 \mathrm{ng}$ of template DNA, $1 \times$ PCR buffer, $2.25 \mathrm{~mm}$ $\mathrm{MgCl}_{2}, 0.1 \mathrm{~mm}$ each of dNTP, $0.4 \mu \mathrm{M}$ RAPD primer (Sangon, Shanghai, China), and 1 unit of Taq DNA polymerase. The mixture was subjected to PCR using the following conditions: 1 cycle of $94{ }^{\circ} \mathrm{C}$ for $3 \mathrm{~min}, 36^{\circ} \mathrm{C}$ for 1 min, $72{ }^{\circ} \mathrm{C}$ for $3 \mathrm{~min}$, and 35 cycles of $94^{\circ} \mathrm{C}$ for $50 \mathrm{~s}, 36^{\circ} \mathrm{C}$ for $50 \mathrm{~s}$, and $72^{\circ} \mathrm{C}$ for $2 \mathrm{~min}$, followed by a final extension of $72{ }^{\circ} \mathrm{C}$ for 7 min. Products were analyzed on $1.4 \%$ agarose gels containing $0.5 \mu \mathrm{g} \cdot \mathrm{mL}^{-1}$ ethidium bromide, and photographed.

Data analysis. The ISSR and RAPD bands were scored as presence (1) or absence $(0)$, and only those that were well defined and consistently repeatable in two independent amplifications were included in the analysis. Similarity matrices based on Jaccard's similarity coefficient was used to construct a UPGMA (unweighted pair group method with arithmetic average) dendrogram. Correlation between the ISSR and RAPD matrices was estimated using the Mantel matrix correspondence test. These analyses were performed using NTSYS-PC version 2.1 (ExeterSoftware, Setauket, NY). Statistical stability of the branches in the dendrogram was estimated by bootstrap analysis with 1000 replicates, using the Free Tree 0.9.1.50 software program (Pavlicek et al., 1999).

To evaluate the discriminatory power of molecular markers, polymorphic information content (PIC) and marker index (MI) were calculated. The PIC value was determined by applying the simplified formula (RoldánRuiz et al., 2000) $\mathrm{PIC}_{i}=2 f_{i}\left(1-f_{i}\right)$, where $f_{i}$ is the percentage of the amplified allele (band present) and $\left(1-f_{i}\right)$ is the frequency of the null allele (band absent) of marker $i$. The MI was determined as a product of PIC and the number of polymorphic bands per assay unit.

An analysis of molecular variance (AMOVA) was used to analyze the partition of molecular variation among wintersweet clones. The Euclidean metric distance of Excoffier et al. (1992) was used in the
AMOVA. The homogeneity of molecular variance analysis (HOMOVA), based on Bartlett's statistic, was also applied to test whether there was significant differentiation between two regions. Bartlett's statistics null distributions were obtained after 1000 permutations. Both AMOVA and HOMOVA were performed using WINAMOVA 1.55 software.

\section{Results}

Intersimple sequence repeat marker analysis. Of 57 ISSR primers tested, 11 showed clear, reproducible band patterns and were chosen for this study. A total of 115 fragments, ranging in size from 280 to $2200 \mathrm{bp}$, were identified, and 90 of them $(78.26 \%)$ were polymorphic. Between 5 (UBC844) to 14 (UBC848) fragments were amplified per primer, with an average of 10.45 bands. The number of polymorphic fragments for each primer varied from 3 (UBC844) to 13 (UBC848), with an average of 8.18. Average PIC and MI were 0.22 and 1.91 respectively.

None of any pair accessions exhibited identical band patterns. The amplified ISSR bands were adequate to discriminate all clones. A 530-bp-long band generated by primer UBC841 was a unique band for H35. In six different cases, there was a unique fragment absent.

The polymorphic bands were used to generate the similarity matrix. Jaccard's similarity coefficient ranged from 0.42 (between $\mathrm{H} 10$ and N25) to 0.96 (between $\mathrm{H} 13$ and $\mathrm{H} 22$ ), with a mean similarity of 0.60 . The similarity coefficients between two pairs of cultivars, $\mathrm{H} 2$ and $\mathrm{H} 5$ (both were named as 'Zhaoxia', 0.87), and H13 and H68 (both were named as 'Acuticoncolor', 0.77) were lower than expected, indicating two cases of homonymy existed.

Random amplified polymorphic DNA marker analysis. Of 180 arbitrary primers screened in this study, 19 were selected because of their ability to produce reproducible and well-defined bands. A total of 165 fragments were generated across the 72 clones, ranging in size from 200 to $2000 \mathrm{bp}$. Of these, $105(63.63 \%)$ were polymorphic. The number of bands produced by each primer varied from 4 (OPC14) to 14 (OPC15 and OPK17), with an average of 8.68 bands. The polymorphic bands ranged from 2 (OPC4) to 12 (OPC15), with an average of 5.53 bands per primer. Average PIC and MI were 0.18 and 1.08 respectively.

Similar to ISSR analysis, the amplified RAPD bands can discriminate all clones analyzed in this study. Some bands were unique to a single genotype. The 1500-bplong band produced by the primer OPK 9 was uniquely identified in $\mathrm{H} 29$, whereas the 1200 bp band of primer OPK14 and the 850-bp band of primer OPC15 were only observed in H46.

Jaccard's similarity coefficient varied between 0.46 (between H25 and H29) and 0.97 (between H13 and H22), with an aver- age of 0.62 . The similarity coefficient between genotype $\mathrm{H} 2$ and $\mathrm{H} 5$ was 0.85 , and between $\mathrm{H} 13$ and $\mathrm{H} 68$ was 0.82 , also indicating the existence of homonymy.

The correlation coefficient between RAPD and ISSR data sets was 0.62 , indicating that the two methods had a positive correlation in this study.

Dendrogram obtained with ISSR and RAPD markers. Because the combined data would give a better coverage of the genome (Siragusa et al., 2006), ISSR and RAPD data sets were combined for UPGMA cluster analysis. Eleven clusters were defined (Fig. 1). The distribution pattern of the 72 clones was coherent with their geographical origins. All the clones of Nanjing origin, with the exception of $\mathrm{N} 25$, were included in clusters 06 and 07 . The other clusters consisted of all the clones from Wuhan plus N25.

In the dendrogram, there were only a few subclusters associated with particular morphological characteristics. For instance, four clones with light-green tepals (H33, H37, $\mathrm{H} 38$, and $\mathrm{HY}$ ) were grouped together at similarity of 0.7 in cluster 02 . Cluster 09 contained six clones (H11, H64, H13, H22, H68, and H44), all having yellow inner tepals. The other clusters were composed of clones with wide phenotypic range.

Analysis of molecular variance and $H O M O V A$. To verify the result that wintersweet clones clustered coherent with their geographical origin, AMOVA and HOMOVA were performed. Analysis of molecular variance provides an estimated $\Phi_{\mathrm{ST}}$ of population differentiation, which is equivalent to an $\mathrm{F}_{\mathrm{ST}}$ statistic when the degree of relatedness among the genetic variants is evaluated (Belaj et al., 2004). The majority of the total genetic diversity $(85.68 \%$ with ISSR data, $86.75 \%$ with RAPD data) was attributed to differences among clones within a geographical region (Table 2). However, a significant $\Phi$ value $\left(\Phi_{\mathrm{ST}}=0.143, P<0.001\right.$, with ISSR data; $\Phi_{\mathrm{ST}}=0.132, P<0.001$, with RAPD data) suggested the existence of differentiation between clones from Wuhan and Nanjing. Corresponding HOMOVA also indicated that the molecular variances were heterogeneous between two regions $\left(\mathrm{B}_{\mathrm{p}}=0.049, P=\right.$ 0.039 , with ISSR data; $\mathrm{B}_{\mathrm{p}}=0.217, P<0.001$, with RAPD data).

\section{Discussions}

Genetic diversity. Wintersweet is a dichogamous, predominately outcrossing species. Therefore, a substantial level of genetic diversity was detected among cultivated germplasm (average ISSR-based and RAPD-based genetic similarity was 0.60 and 0.62 respectively). When AMOVA was used to analyze the partition of molecular variation, both ISSR and RAPD data detected a high genetic differentiation among clones within each region $(85.68 \%$ with ISSR data, $86.75 \%$ with RAPD data). This higher variability within a population agrees well with the allogamous nature of wintersweet. Similar results were also obtained in natural 
wintersweet populations (Chen et al., 1999), and cultivar collections of olive (Belaj et al., 2004) and sweetpotato (Zhang et al., 1998).

Nevertheless, significant $\Phi_{\mathrm{ST}}$ and HOMOVA values showed divergence to some extent between the clones of Nanjing and Wuhan. The differentiation may be explained by different selection criteria taken by gardeners for different traits. The result indicates that the two regions harbor different germplasm resources for widening the genetic base in future breeding, and a greater

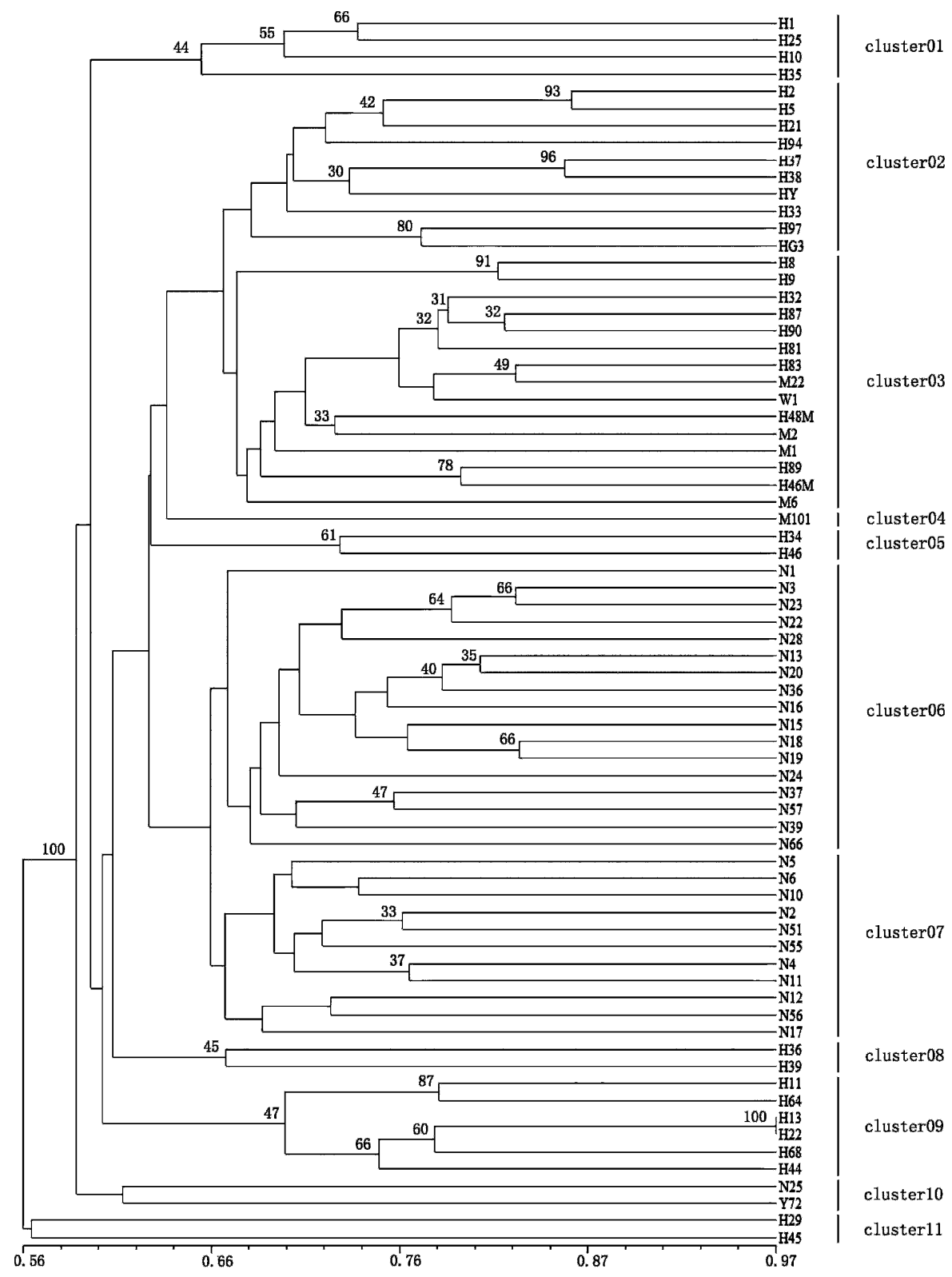

Fig. 1. Dendrogram derived from an unweighted pair group method with arithmetic average (UPGMA) cluster analysis of 72 wintersweet clones based on Jaccard's similarity coefficient matrix, using intersimple sequence repeat marker and random amplified polymorphic DNA marker data. The numbers at the nodes indicate percent bootstrap values of the dendrogram (branches lacking the value received less than $30 \%$ bootstrap support). effort should be taken at collecting and hybridizing wintersweet cultivars from both regions.

Discrimination and classification. Morphological traits, like medium tepal color, inner tepal color, tepal shape, flower shape, and flower size are usually used to identify wintersweet cultivar. Nevertheless, these traits displayed a continuous phenotypic range and were inferred as quantitative traits dominated by multigenes (Chen et al., 1990). In addition, morphological characteristics are considerably affected by environmental factors. Therefore, accurate identification based merely on morphological traits is difficult. For example, $\mathrm{H} 2$ and $\mathrm{H} 5$ (both were named as 'Zhaoxia'), H13 and H68 (both were named as 'Acuticoncolor'), H37 and H38, and H34 and $\mathrm{H} 45$ are difficult to separate based on morphological characteristics. However, in this study, using ISSR or RAPD, they can be easily distinguished. The situation reinforces the importance of using molecular markers for precise identification. Moreover, some fragments were uniquely amplified or absent in a single accession. These fragments are of great interest in optimal management and identification of germplasm collections.

In the existing classification of wintersweet cultivars (Chen et al., 2004; Feng et al., 1990; Zhao et al., 1993), special emphasis was put on the inner tepal color, ranging from yellow, yellow sprinkled with a few mauve speckles, yellow sprinkled with mauve blotches, to mauve. However, the current study demonstrated that although all clones in cluster 09 had yellow inner tepals, in general, clones with the same inner tepal color were dispersed into all groups. Based on morphological cluster analysis, Zhao et al. (2004) found a similar result. In a progeny test, $\mathrm{Wu}(1992)$ found that $3.6 \%$ to $25.0 \%$ of the offspring of individuals with yellow inner tepals had inner tepals with more or less mauve blotches, whereas $5.9 \%$ to $44.4 \%$ of the offspring of individuals with mauve inner tepals had yellow inner tepals. In this study, clones with the same inner tepal color being clustered into different groups in the dendrogram agreed with these previous results, indicating that clones with similar inner tepal color are genetically heterogeneous.

Furthermore, middle tepals of wintersweet showed different colors, from whitish yellow to greenish yellow to yellow to golden. Zhao et al. (1993) proposed the middle tepal color as the first grade of classification criterion. In the current study, clones were not clustered according to flower color,

Table 2. Analysis of molecular variance and homogeneity of molecular variance analysis for the partitioning of intersimple sequence repeat marker (ISSR) and random amplified polymorphic DNA marker (RAPD) variation in wintersweet clones between and within regions (Wuhan and Nanjing).

\begin{tabular}{|c|c|c|c|c|c|c|c|c|}
\hline & $\begin{array}{l}\text { Source of } \\
\text { variation }\end{array}$ & $\mathrm{df}$ & $\begin{array}{l}\text { Variance } \\
\text { components }\end{array}$ & $\begin{array}{c}\text { Total variance } \\
(\%)\end{array}$ & $\Phi$-statistics & $P$ value & Bartlett's index & $P$ value \\
\hline & Within regions & 70 & 11.99 & 85.68 & & & & \\
\hline
\end{tabular}

ISSR, intersimple sequence repeat markers; RAPD, random amplified polymorphic DNA markers. 
only greenish yellow flower clones (H33, $\mathrm{H} 37, \mathrm{H} 38$ and $\mathrm{HY}$ ) could be grouped together in cluster 02. However, in wintersweet, greenish yellow flower is a very rare characteristic. Only four such clones were collected in this study; their relatively high similarity (0.7) may indicate they were developed from a common ancestor. In the dendrogram, clones with same tepal shapes, flower shapes, or flower sizes were also dispersed in all clusters.

In general, the molecular classifications more or less follow morphological classification. However, in our study, discrepancy exists between morphological characteristics and molecular data. This might be most likely the result of the primary stage of wintersweet's breeding, during which most cultivars and varieties derived from open pollination, artificial selection, and maintained by vegetative propagation. There does not yet exist "breeding pools" intended as germplasm owned by a breeder that is repeated intercrossed within it. In similar studies characterizing olive (Hagidimitriou et al., 2005) and almond cultivars (MirAli and Nabulsi, 2003), very low correlation was found between the molecular data and the morphological data. Based on the result, Hagidimitriou et al. (2005) proposed that morphological traits were not reliable in estimating genetic relationships among large and diverse groups of cultivars.

Amplification of ISSR and RAPD. The reliability of RAPD is widely discussed; nevertheless, in our study, PCR conditions were well set up, and only well-defined fragments were included in analyses. Therefore, good reproducibility was achieved with both RAPD and ISSR markers. Many studies have reported that the ISSR technique is highly efficient and reproducible (Arnau et al., 2003; Levi et al., 2004). In this study, higher PIC and MI values were achieved with ISSR than with RAPD, confirming ISSR markers were more informative because of their greater ability to detect polymorphism. Correlation analysis indicated a positive relationship $(r=$ 0.62 ) between ISSR and RAPD; the $r$ value was lower than the study in sour orange $(r=$ 0.93) (Siragusa et al., 2006), but higher than the study in blackgram $(r=0.32)$ (Souframanien and Gopalakrishna, 2004). For two independently derived data sets, a Mantel correlation value $(r)$ greater than 0.5 will be statistically significant at the $0.01 P$ level if the number of observed taxonomic units exceeds 15 (Souframanien and Gopalakrishna, 2004).

Poly (AT) dinucleotide repeats are thought to be the most abundant motifs in plants (Morgantae and Olivieri, 1993); neverthe- less, ISSR primers based on $(\mathrm{AT})_{\mathrm{n}}$ yielded no amplification products in this study. The result is consistent with the findings of Blair et al. (1999) for rice and Moreno et al. (1998) for grapevine. This may indicate that wintersweet genome lacks the $(\mathrm{AT})_{\mathrm{n}}$ motif or that it has very few of these microsatellites.

In conclusion, our results indicate ISSR and RAPD markers are useful for distinguishing and characterizing wintersweet germplasm. The genetic relatedness among these genotypes could provide useful information for conservation and selection of crossparents in breeding.

\section{Literature Cited}

Arnau, G., J. Lallemand, and M. Bourgoin. 2003. Fast and reliable strawberry cultivar identification using inter simple sequence repeat (ISSR) amplification. Euphytica 129:69-79.

Belaj, A., Z. Satovic, I. Trujillo, and L. Rallo. 2004. Genetic relationships of Spanish olive cultivars using RAPD markers. HortScience 39:948951.

Blair, M.W., O. Panaud, and S.R. McCouch. 1999. Inter-simple sequence repeat (ISSR) amplification for analysis of microsatellite motif frequency and fingerprinting in rice (Oryza sativa L.). Theor. Appl. Genet. 98:780-792.

Chen, Z.X. 1995. The study on peroxidase isoenzyme of 17 cultivars of Chimonanthus praecox. Bul. Bot. Res. 15:403-411.

Chen, L.Q. and J.Y. Chen. 1999. The morphology, distribution, classification and utilization of Chimonanthus. J. Chinese Landscape Architecture 15:76-77.

Chen, L.Q., J.Y. Chen, Y.L. Zheng, and D.F. Lu. 1999. Detection of genetic variation within and between natural populations of Chimonanthus praecox (L.) Link using RAPD makers. J. Beijing For. Univ. 21:86-90.

Chen, L.Q., D.F. Lu, and Z.Y. Chen. 1990. Study on wild Chimonanthus praecox in Hubei province. J. Chinese Landscape Architecture 6:24-26.

Chen, L.Q., K.G. Zhao, and M.Q. Zhou. 2004. Cultivar classification system of Chimonanthus. J. Beijing For. Univ. 26(Suppl.):88-90.

Excoffier, L., P.E. Smouse, and J.M. Quattro. 1992. Analysis of molecular variance inferred from metric distances among DNA haplotypes: Application to human mitochondrial DNA restriction site. Genetics 131:479-491.

Feng, J.E., R.H. Li, D.F. Lu, and Y.Q. Chen. 1990. Wintersweet, p. 167-169. In: J.Y. Chen and X.K. Cheng (eds.). The book of Chinese flowers. Shanghai Culture Publ., Shanghai.

Hagidimitriou, M., A. Katsiotis, G. Menexes, C. Pontikis, and M. Loukas. 2005. Genetic diversity of major Greek olive cultivars using molecular (AFLPs and RAPDs) markers and morphological traits. J. Amer. Soc. Hort. Sci. 130:211-217.
Levi, A., C.E. Thomas, M. Newman, O.U.K. Reddy, X. Zhang, and Y. Xu. 2004. ISSR and AFLP markers differ among American watermelon cultivars with limited genetic diversity. J. Amer. Soc. Hort. Sci. 129:553-558.

MirAli, N. and I. Nabulsi. 2003. Genetic diversity of almonds (Prunus dulcis) using RAPD technique. Sci. Hort. 98:461-471.

Moreno, S., J.P. Martin, and J.M. Ortiz. 1998. Inter-simple sequence repeats PCR for characterization of closely related grapevine germplasm. Euphytica 101:117-125.

Morgantae, M. and A.M. Olivieri. 1993. PCRamplified microsatellites as markers in plant genetics. Plant J. 3:175-182.

Pavlicek, A., S. Hrda, and J. Flegr. 1999. FreeTree: Freeware program for construction of phylogenetic trees on the basis of distance data and bootstrap/jackknife analysis of the tree robustness. Application in the RAPD analysis of genus Frenkelia. Folia Biol. (Praha) 45:97-99.

Roldán-Ruiz, I., E. Calsyn, T.J. Gilliland, R. Coll, M.J.T. Van Eijk, and M. De Loose. 2000. Estimating genetic conformity between related ryegrass (Lolium) varieties. 2. AFLP characterization. Mol. Breed. 6:593-602.

Siragusa, M., F. De Pasquale, L. Abbate, and N. Tusa. 2006. Identification of sour orange accessions and evaluation of their genetic variability by molecular marker analyses. HortScience 41:84-89.

Souframanien, J. and T. Gopalakrishna. 2004. A comparative analysis of genetic diversity in blackgram genotypes using RAPD and ISSR markers. Theor. Appl. Genet. 109:1687-1693.

Suo, Z.L., W.Y. Li, J. Yao, H. Zhang, Z. Zhang, and D. Zhao. 2005. Applicability of leaf morphology and intersimple sequence repeat markers in classification of tree peony (Paeoniceae) cultivars. HortScience 40:329-334.

Williams, J.G., A.R. Kubelik, K.J. Livak, J.A Rafalski, and S.V. Tingey. 1990. DNA polymorphisms amplified by arbitrary primers are useful as genetic markers. Nucl. Acids Res. 18:6531-6535.

$\mathrm{Wu}$, J.Z. 1992. Selection of new cultivars and biological characteristics of Chimonanthus praecox (L.) Link. J. Beijing For. Univ. 14(Suppl.):107-111.

Zhang, D.P., M. Ghislain, Z. Huamán, A. Golmirzaie, and R. Hijmans. 1998. RAPD variation in sweetpotato (Ipomoea batatus (L.) Lam) cultivars from South America and Papua New Guinea. Genet. Resource Crop Ev. 45: 271-277.

Zhao, T.B., Z.X. Chen, B.Z. Gao, Z.Q. Li, and L.G. Song. 1993. Chinese wintersweet. Henan Science and Technology Press, Zhengzhou.

Zhao, K.G., J.J.F. Yu, and L.Q. Chen. 2004. Numerical classification and principal component analysis of wintersweet cultivars. J. Beijing For. Univ. 26(Suppl.):79-83.

Zietkiewicz, E., A. Rafalski, and D. Labuda. 1994. Genome fingerprinting by simple sequence repeat (SSR)-anchored polymerase chain reaction amplification. Genomics 20:176-183. 